\title{
Use of a novel Screen-Enrich-Combine(-
}

biomaterials) Circulating System for filling of a 3Dprinted open Ti6Al4V frame with mesenchymal stem cells/ $\beta$-tricalcium phosphate to repair complex anatomical bone defects in load-bearing areas

\section{Wenxiang Chu}

Shanghai Key Laboratory of Orthopaedic Implants, Department of Orthopaedic Surgery, Shanghai Ninth People's Hospital, Shanghai Jiao Tong University School of Medicine, Shanghai 200011, China.

Department of Orthopedic Surgery, Changzheng Hospital, Second Mi

Zhiqing Liu

Shanghai Key Laboratory of Orthopaedic Implants, Department of Orthopaedic Surgery, Shanghai Ninth People's Hospital, Shanghai Jiao Tong University School of Medicine, Shanghai 200011, China

\section{Yaokai Gan ( $\nabla$ ganyk2004@126.com )}

Shanghai Jiao Tong University School of Medicine

\section{Yongyun Chang}

Shanghai Key Laboratory of Orthopaedic Implants, Department of Orthopaedic Surgery, Shanghai Ninth People's Hospital, Shanghai Jiao Tong University School of Medicine, Shanghai 200011, China

Xin Jiao

Shanghai Key Laboratory of Orthopaedic Implants, Department of Orthopaedic Surgery, Shanghai Ninth People's Hospital, Shanghai Jiao Tong University School of Medicine, Shanghai 200011, China

\section{Wenbo Jiang}

Shanghai Key Laboratory of Orthopaedic Implants, Department of Orthopaedic Surgery, Shanghai Ninth People's Hospital, Shanghai Jiao Tong University School of Medicine, Shanghai 200011, China

\section{Kerong Dai}

Shanghai Key Laboratory of Orthopaedic Implants, Department of Orthopaedic Surgery, Shanghai Ninth People's Hospital, Shanghai Jiao Tong University School of Medicine, Shanghai 200011, China

\section{Research}

Keywords: 3D printing, enrichment technique, mesenchymal stem cell, biomaterial, bone repair. 
DOI: https://doi.org/10.21203/rs.3.rs-64338/v1

License: (c) (i) This work is licensed under a Creative Commons Attribution 4.0 International License. Read Full License 


\section{Abstract}

Background: Repairing complex anatomical load-bearing bone defects is difficult because it requires restoration of load-bearing function, reconstruction of anatomical shape and repair by regenerated bone. We previously developed a Screen-Enrich-Combine(-biomaterials) Circulating System (SECCS) for rapid intraoperative enrichment of autologous bone marrow mesenchymal stem cells (MSCs) to enhance the osteogenic ability of porous bone substitutes. In this study, we prepared a 3D-printed Ti6A14V macroporous frame matching the defect shape to provide early load-bearing support and evaluated the efficacy of filling the frame with SECCS-processed MSCs/beta tricalcium phosphate ( $\beta$-TCP) for long-term bone growth.

Method: Mechanical testing of the cylindrical Ti6Al4V frame identified optimal 3D printing parameters. The lateral part of a goat distal femur was used as the defect model, and a matching electron beam melting technology-prepared (EBM) Ti6Al4V frame was fitted. Three frames filled with nothing, pure porous $\beta$-TCP or SECCS-processed MSCs/ $\beta$-TCP were fixed onto the defect site. Repair efficacy was evaluated by X-ray radiography, computed tomography, histology and histomorphology.

Results: In the basic regular hexagon printing unit, the combined side width (w) and inscribed circle diameter (d) determines the printing frame's mechanical strength. The compressive load was significantly higher for $w=1.9 \mathrm{~mm}, \mathrm{~d}=4.4 \mathrm{~mm}$ than for $w=1.7 \mathrm{~mm}, \mathrm{~d}=4.0 \mathrm{~mm}$ or $\mathrm{w}=2.0 \mathrm{~mm}, \mathrm{~d}=5.0 \mathrm{~mm}(\mathrm{P}<$ 0.05). The EBM-prepared Ti6Al4V defect-matched frame was well maintained 9 months after implantation. The MSCs successfully adhered to the wall of the porous $\beta$-TCP in the SECCS-processed group and had spread fully in the test samples. Each goat in the MSCs/ $\beta-T C P-$ filling group had approximately $31,321.7 \pm 22,554.7 \mathrm{MSC}$ and a larger area of new bone growth inside the frame than the areas in the control and blank groups.

Conclusion: Filling the 3D-printed Ti6Al4V large-aperture frame with osteogenic materials achieved biological reconstruction over a larger area of regenerated bone for repair of complex anatomical weightbearing bone defects under the condition of early frame-supported load bearing. MSCs/ $\beta$-TCP prepared by SECCS can be used as a filling material for this type of bone defect to obtain more efficacious bone repair.

\section{Background}

Complex defects in weight-bearing parts of bone are often observed in clinical settings that perform pelvic reconstructions; repairs of bone defects in the acetabulum, femoral condyle and proximal tibia caused by tumours and operations in patients with severe trauma, infection and prosthesis-related osteolysis [1-3]. Repairs of such complex bone defects are difficult because they require restoration of load-bearing function, reconstruction of anatomical shape and repair by regenerated bone, so it has great clinical challenges. Current treatments focus on one or two of these three tasks since it can be difficult to repair all at once. For example, structural bone grafts as part of bone repair cannot tolerate early weight- 
bearing and complex morphological reconstruction and metal products, such as pads, cups and cages, used to provide weight-bearing during postoperative recovery, only allow a very limited volume of autologous bone growth [4-6]. Consequently, providing early weight-bearing function in bone reconstruction that allows the maximum volume of new bone growth inside a complex anatomically shaped support frame would be the major goal of such defect repair.

The increasing capabilities of 3D printing has enabled more accurate and personalised reconstruction of complex anatomical structures. Although a 3D-printed microporous structure can permit a certain degree of bone ingrowth, the volume of in-grown bone is still very limited. To achieve maximal reconstruction by new bone, a 3D-printed large-aperture metal frame providing basic mechanical support that is filled with osteogenic materials inside the frame to achieve long-term regenerated bone filling is currently a more feasible solution. At present, the insufficient amount of autologous bone and limited osteogenic ability of conventional bone substitutes have led to research efforts aimed at identifying bone fillings with excellent osteogenic properties [7-9]. The stem cell Screen-Enrich-Combine(-biomaterials) Circulating System (SECCS), developed previously by us, can rapidly prepare enriched mesenchymal stem cells (MSCs)/beta tricalcium phosphate ( $\beta$-TCP) composites, which have shown good bone repair ability in animal and various clinical bone defect models $[10,11]$. However, the efficacy of repair needs to be further verified as an in-frame filling to repair complex anatomical bone defects.

In this study, we used the lateral half of a goat distal femur as a defect model to assess the ability of 3Dprinted large-aperture titanium alloy frames filled with MSCs/ $\beta$-TCP prepared by SECCS to repair complex anatomical weight-bearing bone defects with the goal of providing a new mode of treatment for such bone defects.

\section{Methods}

\section{General experimental design}

All experimental animal procedures were approved by the Animal Ethics Committee of the Shanghai Ninth People's Hospital. Fifteen goats $(40-45 \mathrm{~kg}$ ) were included and randomly assigned in this study to the experimental group $(n=5)$ that underwent repair with a macroporous titanium alloy (Ti6Al4V) frame filled with SECCS-processed MSCs/ $\beta-T C P$, the control group $(n=5)$ that underwent repair with a Ti6AI4V frame filled with pure $\beta$-TCP and a blank group $(n=5)$ that underwent repair with an unfilled Ti6Al4V frame. The general experimental protocol is shown in Fig. 1.

\section{Parameter optimisation and mechanical strength verification for the 3D-printed Ti6Al4V frame}

A cylindrical testing frame (2.5-cm diameter * 5 - $\mathrm{cm}$ height) with a basic regular hexagon unit was designed by using Mimics software (Materialise, Belgium). The width of this hexagon's sides ( $w$ ) and the diameter of the circle inscribed in it (d) were used as the adjustable parameters. Three different combinations of this width and diameter, namely $(w=1.7 \mathrm{~mm}, \mathrm{~d}=4.0 \mathrm{~mm}),(w=1.9 \mathrm{~mm}, \mathrm{~d}=4.4 \mathrm{~mm})$ and $(w=2.0 \mathrm{~mm}, d=5.0 \mathrm{~mm})$, were used to fabricate the cylindrical Ti6Al4V testing frames by using electron beam melting (EBM) (Fig. 2a). A mechanical testing machine (Instron 8874; Instron, USA) was used to 
perform compression tests to compare the mechanical properties of the three frames. The parameter combination with the best mechanical performance was used to prepare the frames used in the animal model.

\section{Fabrication of Ti6Al4V frame and rounding of its distal surface}

Computed tomography (CT) data of the goats' right-hind knees were input into Mimics software to construct frames matched to the lateral half of the distal femurs in the study goats. The basic regular hexagon unit had a side width of $1.9 \mathrm{~mm}$ and an inscribed circle diameter of $4.4 \mathrm{~mm}$ (Figs. 3a-c). At the same time, the fixing plate and screw position on the frame were also designed. The designed frame and fixing system were fabricated with Ti6Al4V by EBM (Figs. 3d-f). To round the distal surface of the frame, a poly ( $\varepsilon$-caprolactone) (PCL) membrane with a thickness of $2 \mathrm{~mm}$ and a line spacing of approximately $50 \mu \mathrm{m}$ was prepared by fused deposition modelling. Then, the prepared PCL membrane was softened in a $58^{\circ} \mathrm{C}$ water bath and attached to the distal surface of the Ti6Al4V frame (Figs. $3 \mathrm{~g}-\mathrm{i}$ ).

\section{Preparation and characterisation of MSCs/ $\beta$-TCP}

The mixture of MSCs/ $\beta$-TCP was prepared by SECCS, as previously reported [10]. Briefly, after the goat was fully anaesthetised, approximately $55 \mathrm{ml}$ of bone marrow was taken from the bilateral anterior superior iliac spines. Two $\mathrm{ml}$ of bone marrow was taken for nucleated cells and colony-forming units that express alkaline phosphatase (CFU/ALP+) counting. The remaining bone marrow and $6 \mathrm{~g}$ of porous $\beta$ TCP (1-3 mm diameter, $75 \%$ porosity) were placed in the SECCS for preparation of the MSCs/ $\beta$-TCP composites. After this process, the bone marrow was reserved for the same counting.

To confirm whether MSCs had successfully combined with $\beta$-TCP through SECCS, SECCS-processed MSCs/ $\beta$-TCP particle testing samples were cultured in Minimum Essential Medium (a-MEM) supplemented with $10 \%$ foetal bovine serum at $37^{\circ} \mathrm{C}$ in $5 \% \mathrm{CO}_{2}$ for 2 weeks. Then, the particles in the samples were observed by scanning electron microscopy (SEM) and confocal laser scanning microscope. Before the observation by SEM, the particles were subjected to glutaraldehyde fixation, critical point drying and then metal spraying. For detection by confocal laser scanning microscope, the samples were fixed with $4 \%$ paraformaldehyde and incubated with $5 \%$ Triton- 100 for 5 min, followed by incubation with rhodamine at room temperature in the dark for 40 min and then with 4',6-diamidino-2phenylindole for 5 min.

\section{Establishment and repair of bone defect model}

All animals were fasted for $24 \mathrm{~h}$ prior to anaesthesia. General anaesthesia was induced via an intravenous injection of xylazine $(0.15 \mathrm{~mL} / \mathrm{kg})$ and $3 \%$ pentobarbital sodium $(10 \mathrm{~mL})$ and maintained with $3 \%$ pentobarbital sodium (3-5 mL) according to the goats' condition. Then, endotracheal intubation and electrocardiographic monitoring were performed. After bone marrow aspiration, each goat's knee was fully exposed and the lateral half of the distal femur removed using an electric saw to establish the bone defect model. At the same time, MSCs/ $\beta$-TCP particles prepared by SECCS were filled into the Ti6AI4V 
frame, with a Bio-gide membrane sutured into the surface of the PCL membrane to further protect the joint. Then, the processed frame was fixed at the defect site by using a customised plate and screws.

\section{Replanted cell counting}

For nucleated cell counting, the precipitate from $1 \mathrm{ml}$ of pre- or post-SECCS-processed bone marrow after centrifugation was mixed with red blood cell lysate (TBD, China) to remove red blood cells, and the

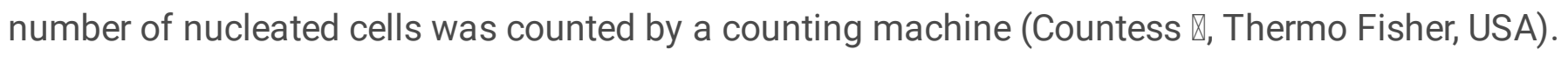

To count the number of MSCs, $1 \mathrm{ml}$ of pre- or post-SECCS-processed bone marrow was diluted in the basic medium and seeded into six-well plates at a density of $0.1 \mathrm{ml} /$ well. After 2-days culturing, proosteogenic medium (a-MEM supplemented with 10\% foetal bovine serum, $0.1 \mathrm{mM}$ dexamethasone, $50 \mathrm{mM}$ ascorbic acid and $10 \mathrm{mM} \beta$-glycerophosphate sodium) was added and changed every 2 days at $37^{\circ} \mathrm{C}$ with $5 \% \mathrm{CO}_{2}$ for osteogenesis of MSCs. Two weeks later, alkaline phosphatase staining was performed, as previously reported, to count the number of CFU/ALP + as the number of MSCs.

The replanted cell number was calculated according to the following formula:

$\mathrm{N}=\mathrm{N}_{\text {pre }} * \mathrm{~V}_{\text {pre }}-\mathrm{N}_{\text {post }} * \mathrm{~V}_{\text {post }}$

where $\mathrm{N}_{\text {pre }}$ and $\mathrm{N}_{\text {post }}$ represent the number of cells (including NCs and MSCs) per ml of bone marrow preand post-SECCS, respectively. $\mathrm{V}_{\text {pre }}$ and $\mathrm{V}_{\text {post }}$ stand for the total volume of bone marrow pre- and postSECCS, respectively.

\section{Evaluations of bone defect repair}

All bone defects were detected by X-ray 1, 3 and 6 months after surgery and by CT scans 6 and 9 months after surgery. At 9 months after surgery, all animals were sacrificed, and the surgical part of the distal femur was completely removed. Those samples were fixed with paraformaldehyde at room temperature. Then, the samples were embedded in polymethyl methacrylate and cut into $100-\mu \mathrm{m}$ thick slices followed by polishing down to $50 \mu \mathrm{m}$ for Van Gieson's picro-fuchsin staining. The ratios of new bone area in the frame to the whole slice area between different groups according to the staining using Image-Pro Plus 6.0 were compared.

\section{Statistical analysis}

All data were analysed by using SPSS 24.0 software (IBM, USA) and presented as the mean \pm standard deviation. The paired t-test was used to compare paired data. Differences between multiple groups of data were compared by one-way analysis of variance and further by the least significant difference t-test, but by Kruskal-Wallis test when equal variance was not assumed. $P<0.05$ was considered to be indicative of statistical significance.

\section{Results}

\section{Optimisation of printing parameters and preparation of repair frames}


The design parameters of the cylindrical testing frame with regular hexagonal basic structural units were optimised, and then the test frames were made with Ti6Al4V. Three columnar frames with different combinations of width (w) and the inscribed circle diameter (d) of the regular hexagonal unit were prepared: $w=1.7 \mathrm{~mm}, \mathrm{~d}=4.0 \mathrm{~mm} ; \mathrm{w}=1.9 \mathrm{~mm}, \mathrm{~d}=4.4 \mathrm{~mm}$ and $w=2.0 \mathrm{~mm}, \mathrm{~d}=5.0 \mathrm{~mm}$. The frame with parameters of $w=1.9 \mathrm{~mm}, \mathrm{~d}=4.4 \mathrm{~mm}$ did not collapse when the load reached $10,000 \mathrm{~N}$, whereas the other two were destroyed before the load reached 10,000 N (Fig. 2b). Specifically, the maximum load was $9553.7 \pm 51.9 \mathrm{~N}$ for the frame with $\mathrm{w}=1.7 \mathrm{~mm}, \mathrm{~d}=4.0 \mathrm{~mm} ; 8693.6 \pm 146.9 \mathrm{~N}$ for the frame with $\mathrm{w}=$ $2.0 \mathrm{~mm} \mathrm{~d}=5.0 \mathrm{~mm}$ and $>10,000 \mathrm{~N}$ for the frame with $\mathrm{w}=1.9 \mathrm{~mm}, \mathrm{~d}=4.4 \mathrm{~mm}$; the maximum load was significantly higher for the frame with the latter combination than those for the frames with the other two parameter combinations $(P<0.05)$ (Fig. 2c).

A Ti6Al4V frame and internal fixation system with parameters of $w=1.9 \mathrm{~mm}$ and $\mathrm{d}=4.4 \mathrm{~mm}$ and that matched the defect of the 5.3-cm long lateral half of the distal femur of the goat (Figs. $3 a-c$ ) was prepared (Figs. $3 d-f)$. The distal end of the frame was covered with a 2-mm thick PCL membrane having an average pore size of $132 \pm 15.6 \mu \mathrm{m}$ (Figs. $3 \mathrm{~g}-\mathrm{i}$ ).

\section{Biological evaluation of MSCs/ $\beta$-TCP prepared by SECCS and counting of replanted cells}

The working SECCS is shown in Fig. 4a, and the porous $\beta$-TCP particles (Fig. 4b) were processed by SECCS to prepare MSCs/ $\beta$-TCP composites (Fig. 4c). After the MSCs/ $\beta$-TCP test samples were cultured in vitro for 2 weeks, the cells were observed to adhere to the inner wall of $\beta$-TCP and to have fully spread (Figs. 4d, e).

After treatment with SECCS, the number of CFU/ALP + in goat bone marrow decreased significantly ( $\mathrm{t}=$ 3.078; $P=0.037$ ) from $848.3 \pm 649.2$ per $\mathrm{ml}$ to $244.0 \pm 212.6$ per $\mathrm{ml}$ (Figs. $5 \mathrm{a}-\mathrm{c}$ ). There was no significant difference in the decrease of bone marrow nucleated cells $(t=1.977, P=0.119)$ from $11.9 \pm 4.1 * 10^{6}$ per $\mathrm{ml}$ to $11.3 \pm 4.5 * 10^{6}$ per $\mathrm{ml}$ (Fig. $5 \mathrm{~d}$ ). In this study, the average number of replanted MSCs for each goat in the MSCs/ $\beta$-TCP-filling group was approximately $31,321.7 \pm 22,554.7$.

\section{Repairing of bone defects in the lateral half of the goats' distal femur}

The previously made 5.3-cm long bone defect in the goat's lateral half of the distal femur and the matching Ti6Al4V frame filled with MSCs/ $\beta$-TCP particles is shown in Fig. 6 . X-ray radiography showed that the sign of particles scattered on the edge of the frame still was seen in the pure $\beta$-TCP-filling group but not in the MSCs/ $\beta$-TCP-filling group at 3 months after the operation. In addition, the sign of bone hyperplasia at the proximal fracture site could be observed at 3 months after surgery in the MSCs/ $\beta$ TCP-filling group, whereas a similar sign could be seen only at 6 months after surgery in the $\beta$-TCPfilling group. The X-ray radiography also confirmed the perfect match between the frame and defect (Fig. 7a). Tissue growth within the frame could be seen by CT in the blank group, pure $\beta$-TCP-filling group and MSCs/ $\beta$-TCP-filling group at 6 and 9 months after surgery (Fig. 7b). 
Histological analysis was performed to evaluate the new bone growth inside the frame and osseointegration between the frame and offside bone. At the junction of the frame and contralateral bone, good osseointegration was seen in the distal section in each group, but osseointegration in the proximal and middle sections was poor. In the blank group, fibrous tissue predominated inside the frame, and only a small amount of new bone was formed. In the $\beta$-TCP-filling group, the $\beta$-TCP particles were almost completely degraded and substituted mainly by fibrous tissue at each section with a small amount of new bone. In the MSCs/ $\beta$-TCP-filling group, the material was completely degraded, and new bone formation with normal trabecular structure was seen in each section. Further, a regenerated Haversian system was also observed (Fig. 8a). Quantitative analysis showed that the relative area of new bone was significantly higher in the MSCs/ $\beta$-TCP-filling group than in the $\beta$-TCP implantation group or blank group at the proximal section $(10.8 \% \pm 6.0 \%$ vs. $3.7 \% \pm 1.7 \%$ vs. $1.4 \% \pm 0.9 \%$, respectively; $F=$ $10.953, P<0.01)$, middle section $(21.6 \% \pm 7.8 \%$ vs. $6.3 \% \pm 2.6 \%$ vs. $3.4 \% \pm 2.1 \%$, respectively; $F=24.098$, $P<0.01)$ and distal section $(41.5 \% \pm 15.5 \%$ vs. $15.8 \pm 11.0 \%$ vs. $9.1 \% \pm 5.3 \%$, respectively; $F=13.558, P<$ 0.01) (Figs. 8b-d).

\section{Discussion}

Early recovery of weight-bearing function and reconstruction of complex geometries by regenerated autologous bone are the key goals of repairing complex anatomical weight-bearing bone defects. The ideal repair is one in which shape-matched materials provide sufficient mechanical support and induce new bone to grow quickly and fully during the degradation process. However, currently used degradable materials do not guarantee mechanical support as they degrade. In this study, to achieve bone defect repair with as much autologous bone growth as possible under the condition of early load bearing, we prepared a macroporous Ti6AI4V frame with a defect-matching shape by 3D printing to provide shortterm mechanical support and filled the frame with osteogenic biomaterial prepared by SECCS to achieve long-term bone repair by regenerated bone. This strategy is a new approach for repair of complex anatomical weight-bearing bone defects with both short-term and long-term benefits.

Additive manufacturing enables design freedom and manufacturing flexibility, so it has unparalleled advantages in the preparation of customised implants [12]. EBM, by virtue of its higher-energy density, has become the main method of additive manufacturing for metal implant fabrication in recent years [13]. Metal implants prepared by EBM have been used in spinal intervertebral fusion cages, upper cervical vertebral and skull reconstruction [14-16]. To enhance the long-term stability of implants, most of those prepared by EBM have had a microporous structure to provide the potential for long-term osseous ingrowth. However, the pore size of the microporous structures reported so far have mostly ranged between $100-500 \mu \mathrm{m}$, and the volume of bone that can grow is very limited [17]. Therefore, the bony holding force produced by this limited new growth mass is restricted. In this study, we tried to replace the widely used microporous structure with a large-aperture frame structure to leave more space for bone growth. To select the appropriate printing parameters to ensure the mechanical strength of the frame, we evaluated the mechanical properties of the cylindrical frame with three sets of parameters according to the mechanical test standard [18]. Inspired by a honeycomb, in which each comb is connected to other 
combs on all six sides and achieves good space utilisation with less material, we used a regular hexagonal basic printing unit. The width of the side and diameter of the inscribed circle of the hexagonal units are the two key factors that affect the overall mechanical properties of the frame. Theoretically, a larger inscribed circle diameter provides more space for bone filling but reduces the mechanical strength, whereas the opposite relationship is observed for the width of the side. In this study, the frame with the combination of $\mathrm{w}=1.9 \mathrm{~mm}$ and $\mathrm{d}=4.4 \mathrm{~mm}$ for the hexagonal units had better mechanical performance, indicating that the mechanical increment caused by the increase in the side width from $1.7 \mathrm{~mm}$ to $1.9 \mathrm{~mm}$ exceeded the mechanical decrement caused by the increase in the inscribed circle diameter from $4.0 \mathrm{~mm}$ to $4.4 \mathrm{~mm}$. Additionally, the mechanical increase by increasing the side width from $1.9 \mathrm{~mm}$ to $2.0 \mathrm{~mm}$ did not exceed the mechanical decrease by increasing the inscribed circle diameter from $4.4 \mathrm{~mm}$ to $5.0 \mathrm{~mm}$. Consequently, we used the optimal width and diameter parameters to prepare the Ti6Al4V frame, which matched the lateral half of the goat distal femur. The corresponding internal fixation system was designed and fabricated at the same time. Since the defect model involved the articular surface, to minimise potential joint damage, a PCL film was used to cover the frame's distal end to improve smoothness and reduce rigidity. In addition, a Bio-gide collagen membrane was sutured into the surface of this PCL film to further protect the joints.

The osteogenic ability of bone fillers is the key to bone defect repair. Since autologous bone grafting, which currently is the gold standard for bone grafting treatment, has some disadvantages, such as limited supply and bone-collecting-related complications, finding bone substitutes with similar osteogenic ability has always been an important goal in orthopaedic research [19]. Bone marrow MSCs are indispensable for bone repair [20,21]. A large number of studies have confirmed that use of MSCs with bone substitutes can effectively promote bone repair effect [22-24]. The SECCS we developed previously can rapidly incorporate processes of screening, enrichment of autologous bone marrow MSCs and generation of composites with porous bone substitutes intraoperatively. These composites have shown superior osteogenic effects in animal models and clinical patients [10,25]. In this study, the number of MSCs in bone marrow was significantly decreased after treatment with SECCS, indicating that MSCs successfully adhered to the porous $\beta$-TCP. From the testing particles, the adhered MSCs were widely distributed on the inner wall of the $\beta$-TCP and fully spread. The number of bone marrow MSCs replanted is an important factor in determining the effect of bone repair [26, 27]. In this study, each goat was replanted with about $31,321.7 \pm 22,554$.7 MSCs on average in the MSCs/ $\beta$-TCP-filling group. In the reported studies on bone tissue engineering, most of the implanted MSCs were obtained through in vitro culture, and the number of transplanted MSCs often reached millions, which was much higher than that in this study [22,23]. In this regard, we believe that SECCS has the following advantages over in vitro culture technology that outweigh its relatively lower number of replants. First, although the number of MSCs expanded in vitro was huge, they originated from a small number of primary cells. In terms of replanted primary MSCs, SECCS showed an overwhelming advantage. Since MSCs will inevitably undergo replicative senescence during in vitro culture, the proliferation and differentiation ability of MSCs will be reduced to a certain extent after expansion, whereas the MSCs replanted by SECCS retain their original ability $[28,29]$. Second, the bone marrow MSCs expanded in vitro experienced sudden changes in 
the living environment after implanting into the body, which potentially could partially inhibit cell viability. However, the cells replanted by SECCS were not cultured in vitro, circumventing this inhibitory factor.

The lateral half of the goat distal femurs had both partial weight-bearing function and complex geometry and was easy to expose and observe. Therefore, it was used as a bone defect model in this study.

Intraoperative photos and postoperative X-rays showed that the frame prepared by 3D printing completely matched the defect shape, and the shape was still well maintained 9 months after surgery. Postoperative $X$-ray radiograms showed that the scattered particles around the frame in the MSCs/ $\beta-T C P-$ filling group had degraded and disappeared by 3 months after surgery, whereas those in the $\beta$-TCP-filling group were still visible at 3 months after surgery, suggesting that the former had a relatively faster degradation rate than that of the latter, which is consistent with the results found in our previous study [30]. In addition, the bony bridging sign at the proximal fracture site was seen earlier in the MSCs/ $\beta$-TCP-filling group than in the $\beta$-TCP-filling group, which may have been related to the recruitment of autologous MSCs by implanted MSCs to promote local bone ingrowth [31, 32]. From the CT results, even though we rounded the distal end of the frame, there was still joint damage, such as patella displacement and joint wear. This finding may be because the end of the frame damaged the outer protective complex during the wear process. Therefore, for defects with joint retention, it may be more appropriate to adopt a frame with a non-porous and polished surface. In terms of defect repair, the CT results showed that tissue ingrowth was present even in the frames without material filling, suggesting that a microporous structure may not be a necessary condition for tissue ingrowth. Because of the interference by metal artefacts, we could not evaluate the tissue composition on the ground of the grey value of the tissue within the frame.

To observe the osteogenesis effect inside the frame, we performed segmented slices of the repaired frame. A better bone construction effect within the frame could be seen at each section in the MSCs/ $\beta$ TCP-filling group, with new trabecular bone tightly integrated with the frame wall and the Harval system under reconstruction, which suggests that the new bone has good plasticity. Generally, the new bone area at the distal section accounts for a relatively large proportion. This characteristic may be due to the larger contact area between the distal part and contralateral normal bone, which results in a more complete local osteogenesis environment. Studies have shown that the degradation cycle of porous $\beta$-TCP is between 6 to 18 months. In this study, the porous $\beta$-TCP with a diameter of $1-3 \mathrm{~mm}$ implanted in the defect region had been almost degraded by 9 months after surgery, which is consistent with previous reports. Interestingly, in the pure $\beta$-TCP-filling group, a small new bone island appeared at the centre of the filling area, which suggests that the new bone formation mode mediated by porous materials did not completely depend on the gradual replacement from the surrounding normal bone to the material pores. The ossification process may also occur independently inside the porous material, possibly through the penetrated bone marrow. Although the $\mathrm{CT}$ results indicated that there was tissue ingrowth in the three groups, the amount of new bone in the blank group and $\beta$-TCP-filling group was very limited, mainly manifested as fibrous scar tissue. Accordingly, the osteogenic capacity of the filler significantly affected the long-term osteogenic effect, and the MSCs/ $\beta$-TCP prepared by SECCS exhibited better osteogenic performance in this defect model. 
In this study, in response to the clinical problem of complex anatomical bone defects in weight-bearing areas, we proposed and assessed an approach to achieving bone repair by providing a larger volume of final autologous bone; this approach involved preparing a large-aperture weight-bearing frame by 3D printing and placement of biomaterials modified by SECCS in the frame to achieve long-term bone filling. This approach may provide a new treatment option for clinical repair of such bone defects.

\section{Conclusion}

Filling a 3D-printed Ti6Al4V large-aperture frame with osteogenic materials provided a higher extent of biological reconstruction for bone defects with complex anatomical shape in the weight-bearing part under the condition of early weight-bearing. MSCS/ $\beta$-TCP prepared by SECCS can be used as a filling material for this type of bone defect to obtain a good bone repair effect.

\section{Abbreviations}

MSCs: mesenchymal stem cells; $\beta$-TCP: $\beta$-tricalcium phosphate; MSCs/ $\beta$-TCP: MSCs and $\beta$-TCP composites; SECCS: stem cell Screen-Enrich-Combine(-biomaterials) Circulating System; EBM: electron beam melting; CT: computed tomography; ALP: alkaline phosphatase; CFU/ALP+: colony-forming units that expressed alkaline phosphatase; SEM: scanning electron microscope; PCL: polycaprolactone

\section{Declarations}

\section{Ethics approval and consent to participate}

All experimental procedures in this study were approved and performed in accordance with the guidelines of the Animal Ethics Committee of Shanghai Ninth People's Hospital (HKDL[2017]405).

\section{Consent for publication}

Not applicable

\section{Availability of data and materials}

All data generated and/or analysed during this study are included in this published article. Any further information can be obtained from the corresponding author on reasonable request.

\section{Competing interests}

The authors declare that they have no competing interests.

\section{Funding}

This work was supported by the Clinical Research Program of 9th People's Hospital, Shanghai Jiao Tong University School of Medicine [JYLJ015] and the Clinical Research Plan of SHDC [16CR3099B], the Class 
IV Peak Subject Program of Shanghai Jiao Tong University School of Medicine (No. GXQ03) and the National Key Research and Development Program of China (2016YFC1102104, 2017YFC1103900).

\section{Author's contributions}

WC and YG contributed to designing and performing the study and preparing the manuscript. $\mathrm{ZL}$ and $\mathrm{XJ}$ contributed to performing the study. YC contributed to data analysis. WJ contributed to 3D printing of testing and repairing frames. YG and KD supervised the study. All authors read and approved the final manuscript.

\section{Acknowledgements}

The authors sincerely thank Lu Shen for instructions in software usage and also acknowledge Wendong Xue for his help with mechanical test.

\section{References}

1. Anract $\mathrm{P}, \mathrm{Biau} \mathrm{D}$, Babinet A,Tomeno B. [Pelvic reconstructions after bone tumor resection]. Bull Cancer. 2014;101(2):184-94I.

2. Migaud $\mathrm{H}$, Common $\mathrm{H}$, Girard J, Huten D,Putman S. Acetabular reconstruction using porous metallic material in complex revision total hip arthroplasty: A systematic review. Orthop Traumatol Surg Res. 2019;105(1s):S53-s61I.

3. Struckmann V, Schmidmaier G, Ferbert T, Kneser U,Kremer T. Reconstruction of Extended Bone Defects Using Massive Allografts Combined with Surgical Angiogenesis: A Case Report. JBJS Case Connect. 2017;7(1):e10l.

4. Du YQ, Liu YP, Sun JY, Ni M,Zhou YG. Reconstruction of Paprosky type IIIB acetabular bone defects using a cup-on-cup technique: A surgical technique and case series. World $\mathrm{J}$ Clin Cases. 2020;8(7):1223-12311.

5. Zhang Z, Zhao J, Huang $X$, Jiang $H, X u$ Z. [Short-term effectiveness of acetabular reconstruction with three-dimensional printed trabecular metal pads in hip revision]. Zhongguo Xiu Fu Chong Jian Wai Ke Za Zhi. 2019;33(12):1516-1520l.

6. Sporer SM, O'Rourke M, Chong P,Paprosky WG. The use of structural distal femoral allografts for acetabular reconstruction. Average ten-year follow-up. J Bone Joint Surg Am. 2005;87(4):760-5I.

7. Costa Mendes L, Sauvigne T,Guiol J. [Morbidity of autologous bone harvesting in implantology: Literature review from 1990 to 2015]. Rev Stomatol Chir Maxillofac Chir Orale. 2016;117(6):388-402I.

8. Seiler JG, 3rd,Johnson J. Iliac crest autogenous bone grafting: donor site complications. J South Orthop Assoc. 2000;9(2):91-71.

9. Busch A, Wegner A, Haversath M,Jäger M. Bone Substitutes in Orthopaedic Surgery: Current Status and Future Perspectives. Z Orthop Unfall. 2020 
10. Chu W, Gan Y, Zhuang Y, Wang X, Zhao J, Tang T, et al. Mesenchymal stem cells and porous betatricalcium phosphate composites prepared through stem cell screen-enrich-combine(-biomaterials) circulating system for the repair of critical size bone defects in goat tibia. Stem Cell Res Ther. 2018;9(1):157l.

11. Zhuang Y, Gan Y, Shi D, Zhao J, Tang T,Dai K. A novel cytotherapy device for rapid screening, enriching and combining mesenchymal stem cells into a biomaterial for promoting bone regeneration. Sci Rep. 2017;7(1):15463I.

12. Martin JH, Yahata BD, Hundley JM, Mayer JA, Schaedler TA,Pollock TM. 3D printing of high-strength aluminium alloys. Nature. 2017;549(7672):365-369I.

13. Parthasarathy J, Starly B, Raman S,Christensen A. Mechanical evaluation of porous titanium (Ti6Al4V) structures with electron beam melting (EBM). J Mech Behav Biomed Mater. 2010;3(3):249591.

14. Wei F, Li Z, Liu Z, Liu X, Jiang L, Yu M, et al. Upper cervical spine reconstruction using customized 3Dprinted vertebral body in 9 patients with primary tumors involving C2. Ann Transl Med. 2020;8(6):3321.

15. Epasto G, Distefano F, Mineo R,Guglielmino E. Subject-specific finite element analysis of a lumbar cage produced by electron beam melting. Med Biol Eng Comput. 2019;57(12):2771-2781I.

16. Francaviglia N, Maugeri R, Odierna Contino A, Meli F, Fiorenza V, Costantino G, et al. Skull Bone Defects Reconstruction with Custom-Made Titanium Graft shaped with Electron Beam Melting Technology: Preliminary Experience in a Series of Ten Patients. Acta Neurochir Suppl. 2017;124:137141 I.

17. Zhang L, Yang G, Johnson BN,Jia X. Three-dimensional (3D) printed scaffold and material selection for bone repair. Acta Biomater. 2019;84:16-33I.

18. Xie XH, Wang XL, Zhang G, He YX, Leng Y, Tang TT, et al. Biofabrication of a PLGA-TCP-based porous bioactive bone substitute with sustained release of icaritin. J Tissue Eng Regen Med. 2015;9(8):961721.

19. Ebraheim NA, Elgafy $H, X u$ R. Bone-graft harvesting from iliac and fibular donor sites: techniques and complications. J Am Acad Orthop Surg. 2001;9(3):210-8I.

20. Lin W, Xu L, Zwingenberger S, Gibon E, Goodman SB,Li G. Mesenchymal stem cells homing to improve bone healing. J Orthop Translat. 2017;9:19-27l.

21. Hernigou P, Poignard A, Beaujean F,Rouard $H$. Percutaneous autologous bone-marrow grafting for nonunions. Influence of the number and concentration of progenitor cells. J Bone Joint Surg Am. 2005;87(7):1430-7l.

22. Liu G, Zhao L, Zhang W, Cui L, Liu W,Cao Y. Repair of goat tibial defects with bone marrow stromal cells and beta-tricalcium phosphate. J Mater Sci Mater Med. 2008;19(6):2367-76I.

23. Viateau V, Guillemin G, Bousson V, Oudina K, Hannouche D, Sedel L, et al. Long-bone critical-size defects treated with tissue-engineered grafts: a study on sheep. J Orthop Res. 2007;25(6):741-91. 
24. Gan Y, Dai K, Zhang P, Tang T, Zhu Z,Lu J. The clinical use of enriched bone marrow stem cells combined with porous beta-tricalcium phosphate in posterior spinal fusion. Biomaterials. 2008;29(29):3973-3982I.

25. Wang X, Chu W, Zhuang Y, Shi D, Tao H, Jin C, et al. Bone Mesenchymal Stem Cell-Enriched betaTricalcium Phosphate Scaffold Processed by the Screen-Enrich-Combine Circulating System Promotes Regeneration of Diaphyseal Bone Non-Union. Cell Transplant. 2019;28(2):212-223I.

26. Wu H, Kang N, Wang Q, Dong P, Lv X, Cao Y, et al. The Dose-Effect Relationship Between the Seeding Quantity of Human Marrow Mesenchymal Stem Cells and In Vivo Tissue-Engineered Bone Yield. Cell Transplant. 2015;24(10):1957-68I.

27. Petite $H$, Viateau V, Bensaid W, Meunier A, de Pollak C, Bourguignon $M$, et al. Tissue-engineered bone regeneration. Nat Biotechnol. 2000;18(9):959-63I.

28. Sethe S, Scutt A,Stolzing A. Aging of mesenchymal stem cells. Ageing Res Rev. 2006;5(1):91-116l.

29. Ksiazek K. A comprehensive review on mesenchymal stem cell growth and senescence. Rejuvenation Res. 2009;12(2):105-16l.

30. Chu W, Wang X, Gan Y, Zhuang Y, Shi D, Liu F, et al. Screen-enrich-combine circulating system to prepare MSC/beta-TCP for bone repair in fractures with depressed tibial plateau. Regen Med. 2019

31. Baraniak PR,McDevitt TC. Stem cell paracrine actions and tissue regeneration. Regenerative medicine. 2010;5(1):121-143|.

32. Seebach E, Freischmidt H, Holschbach J, Fellenberg J,Richter W. Mesenchymal stroma cells trigger early attraction of M1 macrophages and endothelial cells into fibrin hydrogels, stimulating long bone healing without long-term engraftment. Acta biomaterialia. 2014;10(11):4730-4741I.

\section{Figures}




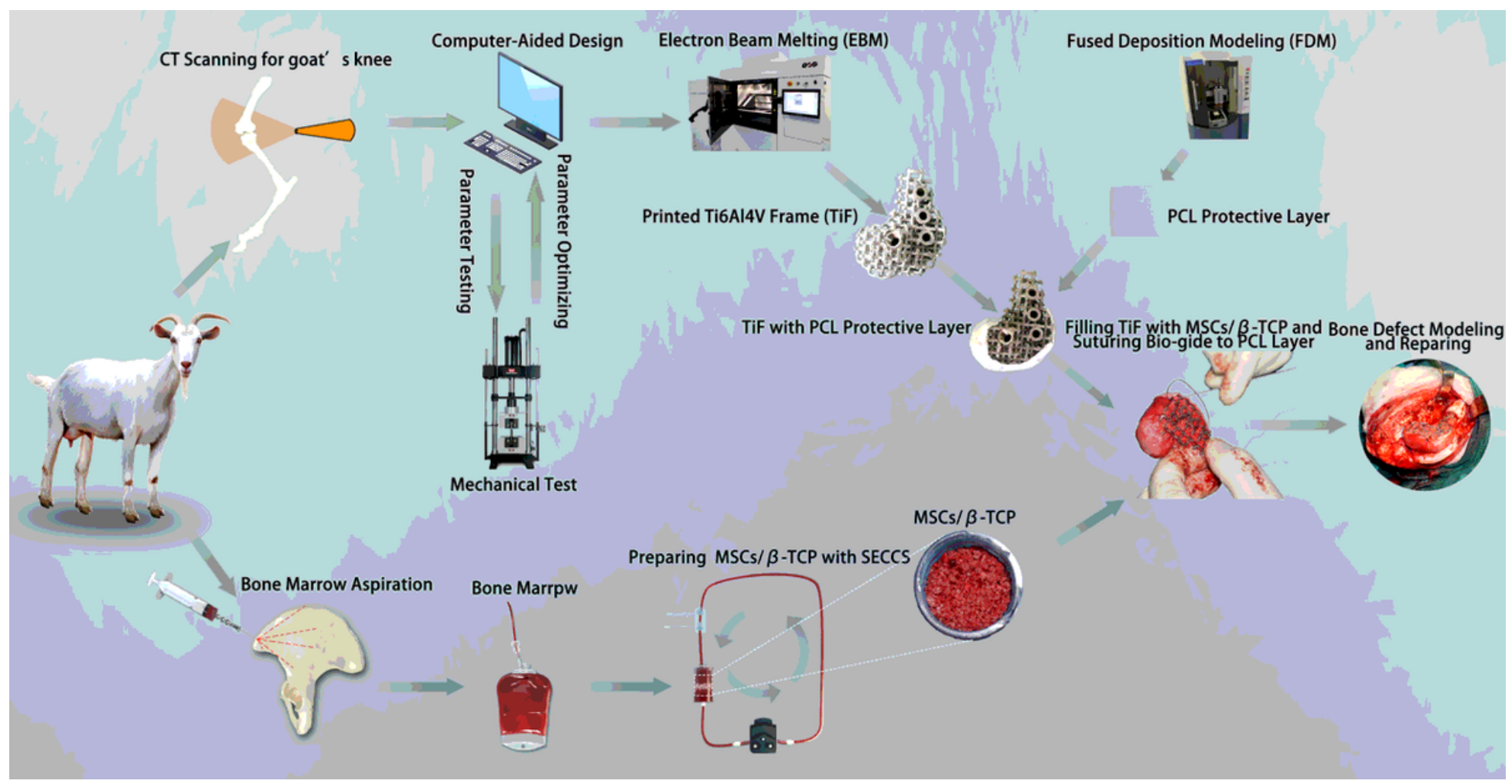

Figure 1

Experimental protocol for repair of bone defects in the goats' lateral half of the distal femur. 
A
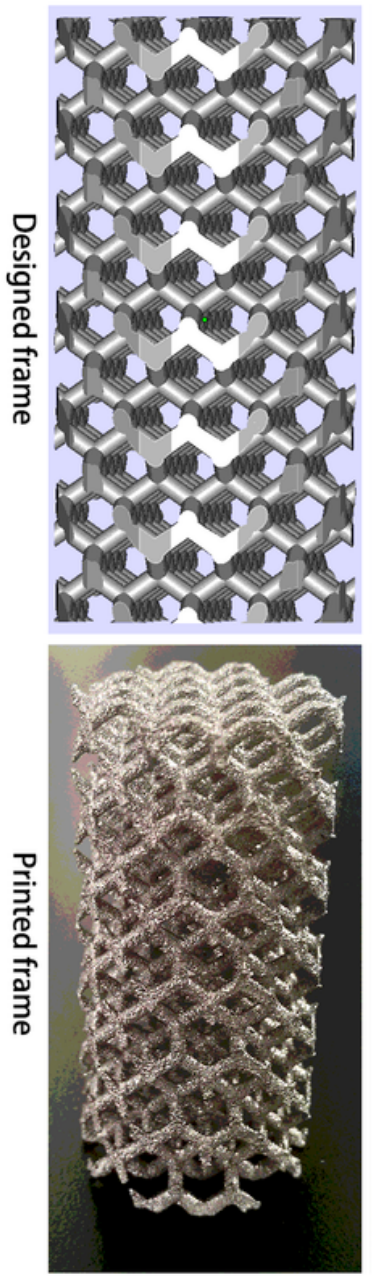

$1.9-4.4$
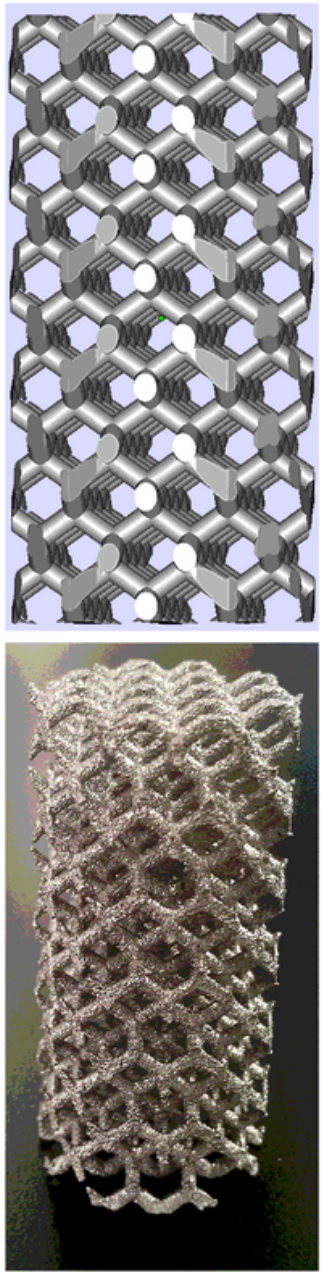

$2.0-5.0$
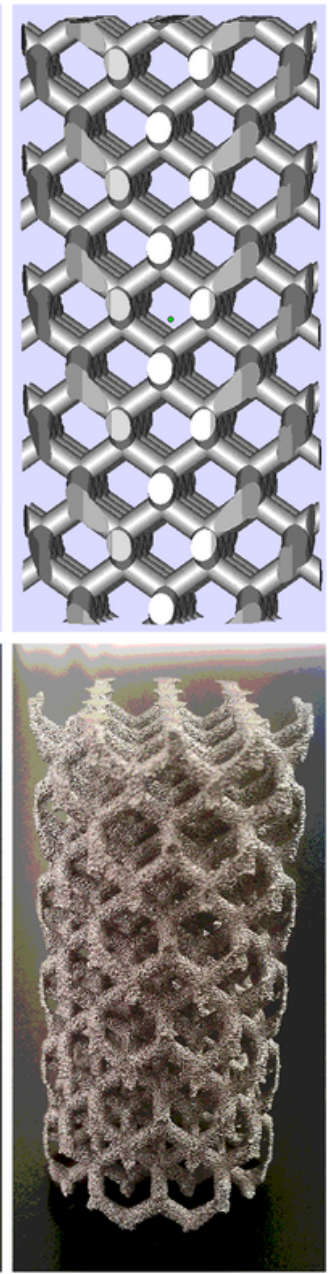
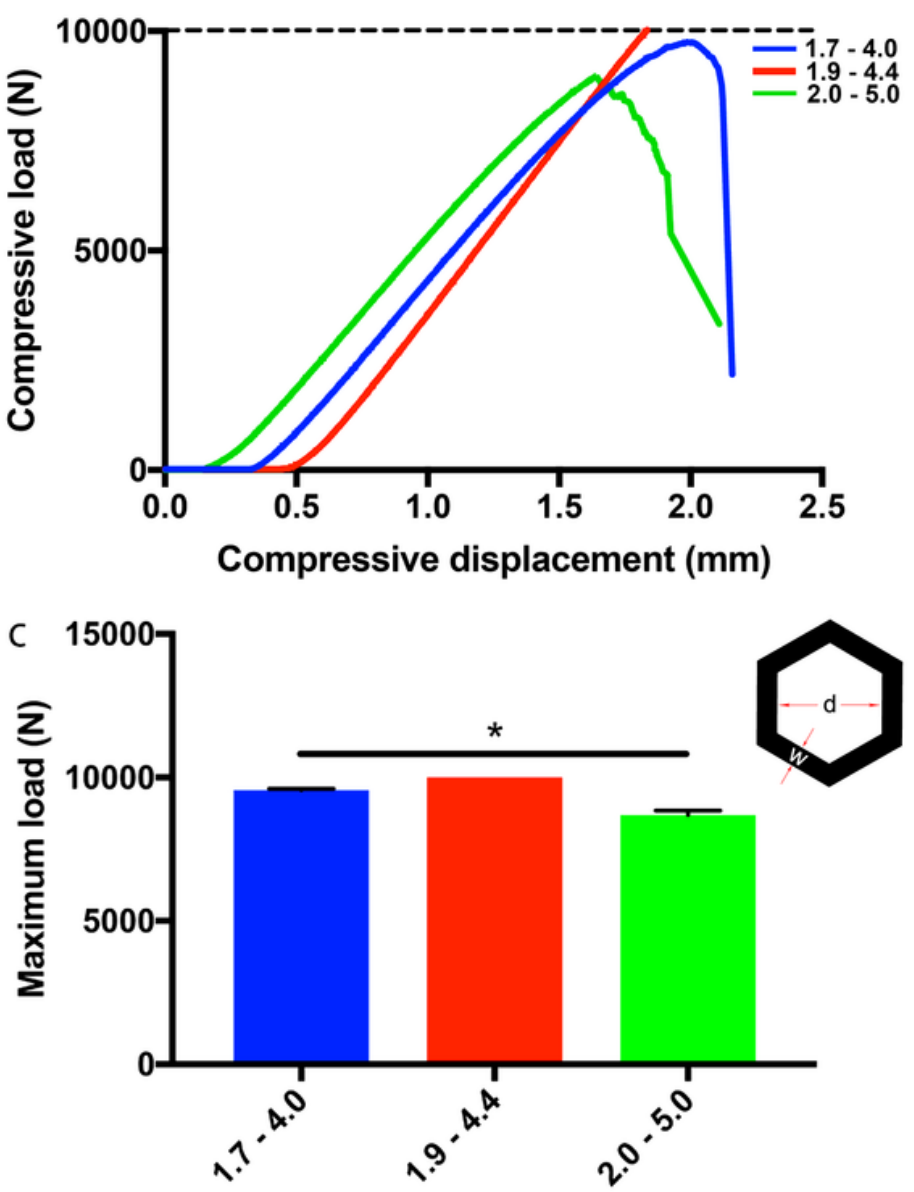

Frame parameter (width - diameter; $\mathbf{m m}$ )

Figure 2

Parameter optimisation by mechanical testing for 3D-printing of the Ti6Al4V open frame. $(a, b)$ Cylindrical frames with hexagonal unit parameters of different side widths and inscribed circle diameters were designed (a) and fabricated with Ti6Al4V (b). (c) The frame with a 1.9-mm width and a 4.4-mm diameter withstood the machine's extreme load of $10,000 \mathrm{~N}$, and the frames with a 1.7-mm width, 4.0-mm diameter and a 2.0-mm width, 5.0- $\mathrm{mm}$ diameter collapsed before the extreme loading. (d) Comparison of maximum loads between frames with different parameters. (Note: the maximum load of the frame with a 1.9-mm width and a 4.4-mm diameter exceeded the upper limit of the machine and was regarded as having the machine's maximum load of $10,000 \mathrm{~N}$.) 

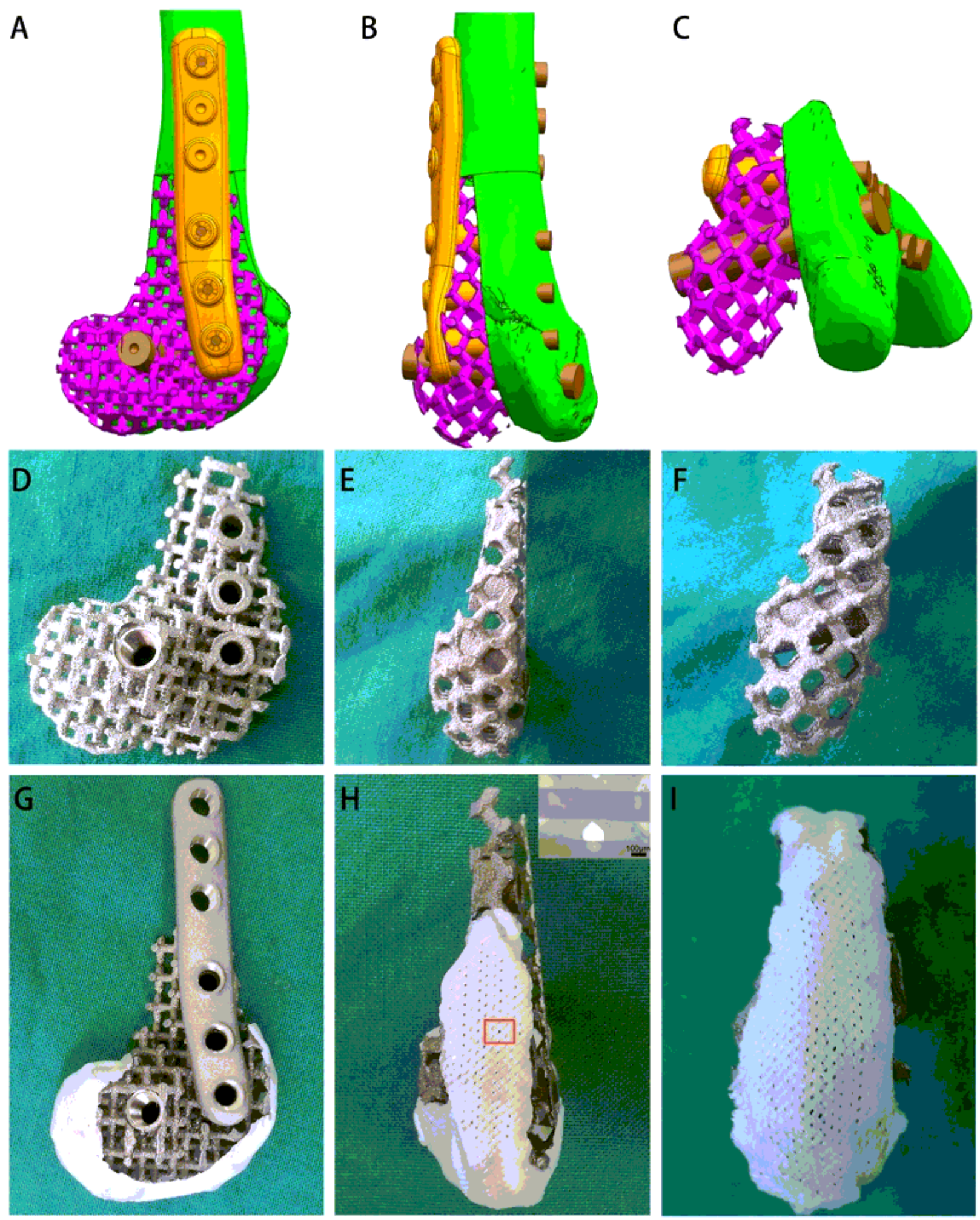

\section{Figure 3}

Design and fabrication of the frame matched with the goat's lateral half of the distal femur. $(a-c)$ Designed frame with inner fixation system in the lateral (a), anteroposterior (b) and axial views (c). (d-f) Fabricated Ti6Al4V frame in the lateral (d), anteroposterior (e) and axial views (f). (g-i) A PCL membrane was attached to the distal surface of the fabricated frame. 

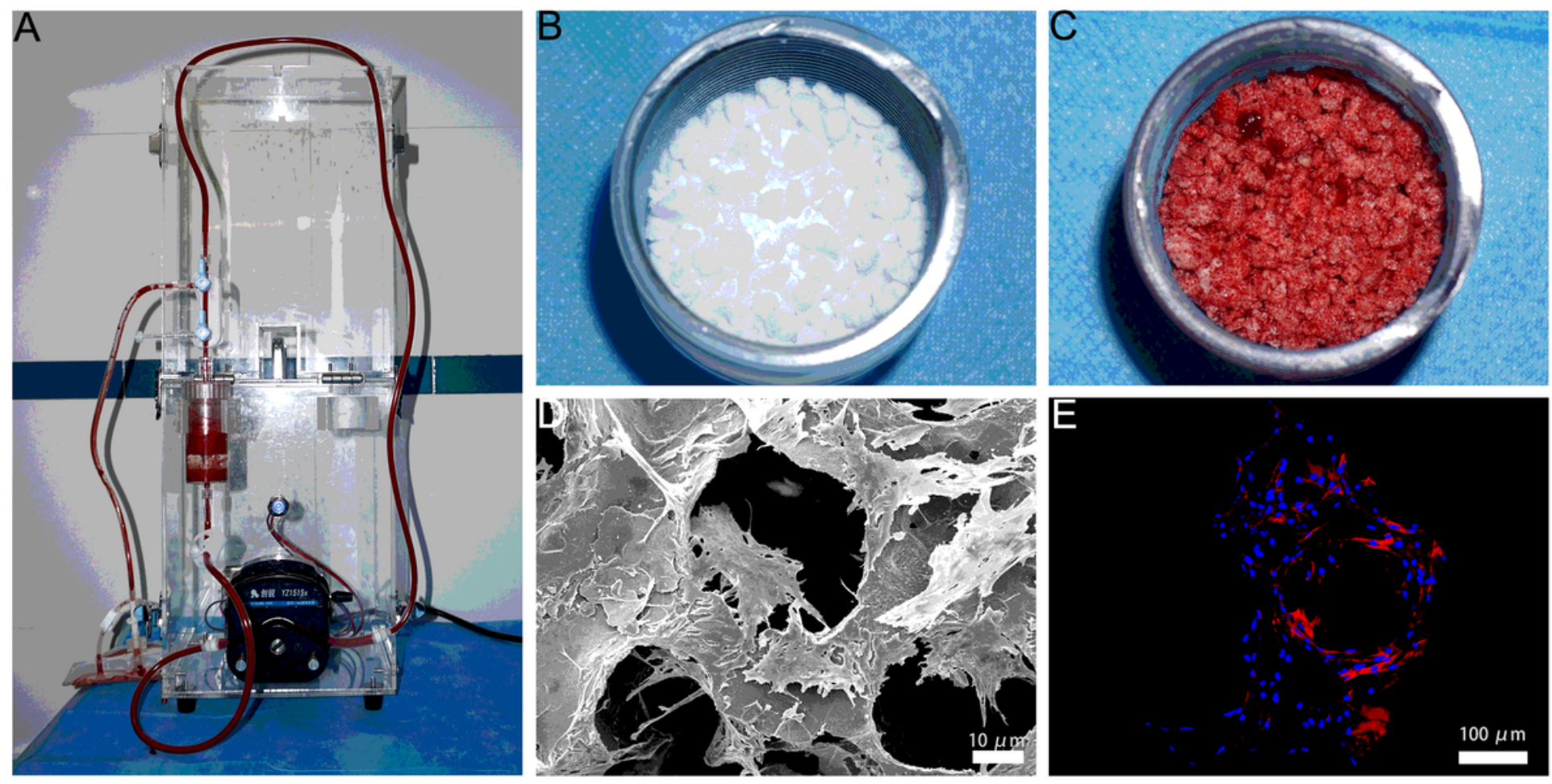

Figure 4

Preparation and characterisation of MSCs/ $\beta$-TCP. (a) The proprietary Screen-Enrich-Combine(biomaterials) Circulating System (SECCS) was used to prepare MSCs/ $\beta$-TCP. (b, c) Porous $\beta$-TCP (b) was transformed into MSCs/ $\beta$-TCP (c) by SECCS. (d, e) Scanning electron microscopy and laser scanning confocal microscopy views showing that the cells adhered to and widely spread on the inner wall of the MSCs/ $\beta$-TCP particle testing samples after 14-day culture. 


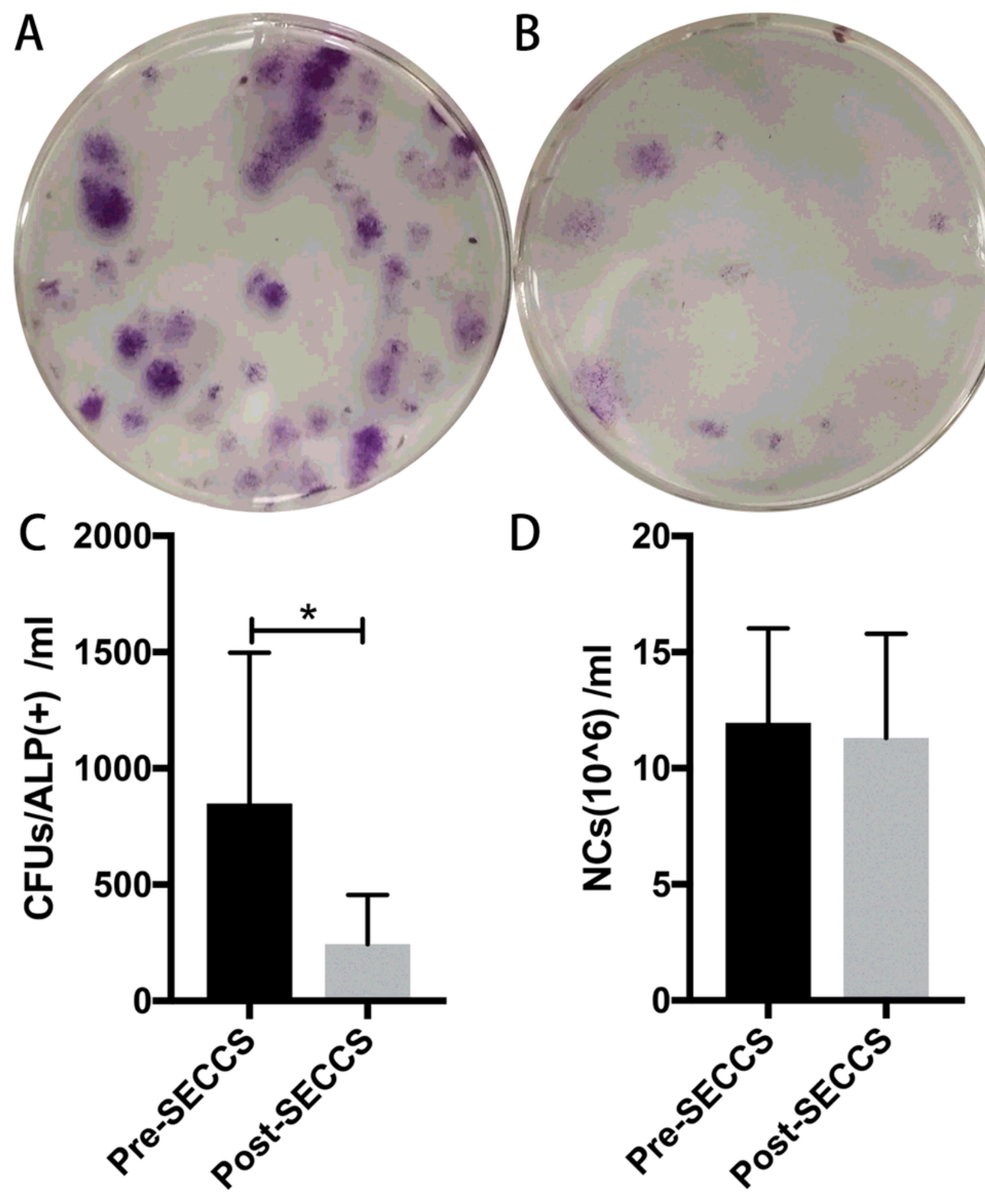

Figure 5

Replanted cell counting. $(a, b)$ CFU/ALP+ counts from bone marrow before (a) and after (b) SECCS processing were obtained. (c, d) The number of CFU/ALP+ (c) or NCs (d) was reduced remarkably after SECCS processing. CFU/ALP+: alkaline phosphatase-positive colony-forming units; NCs: nucleated cells. 

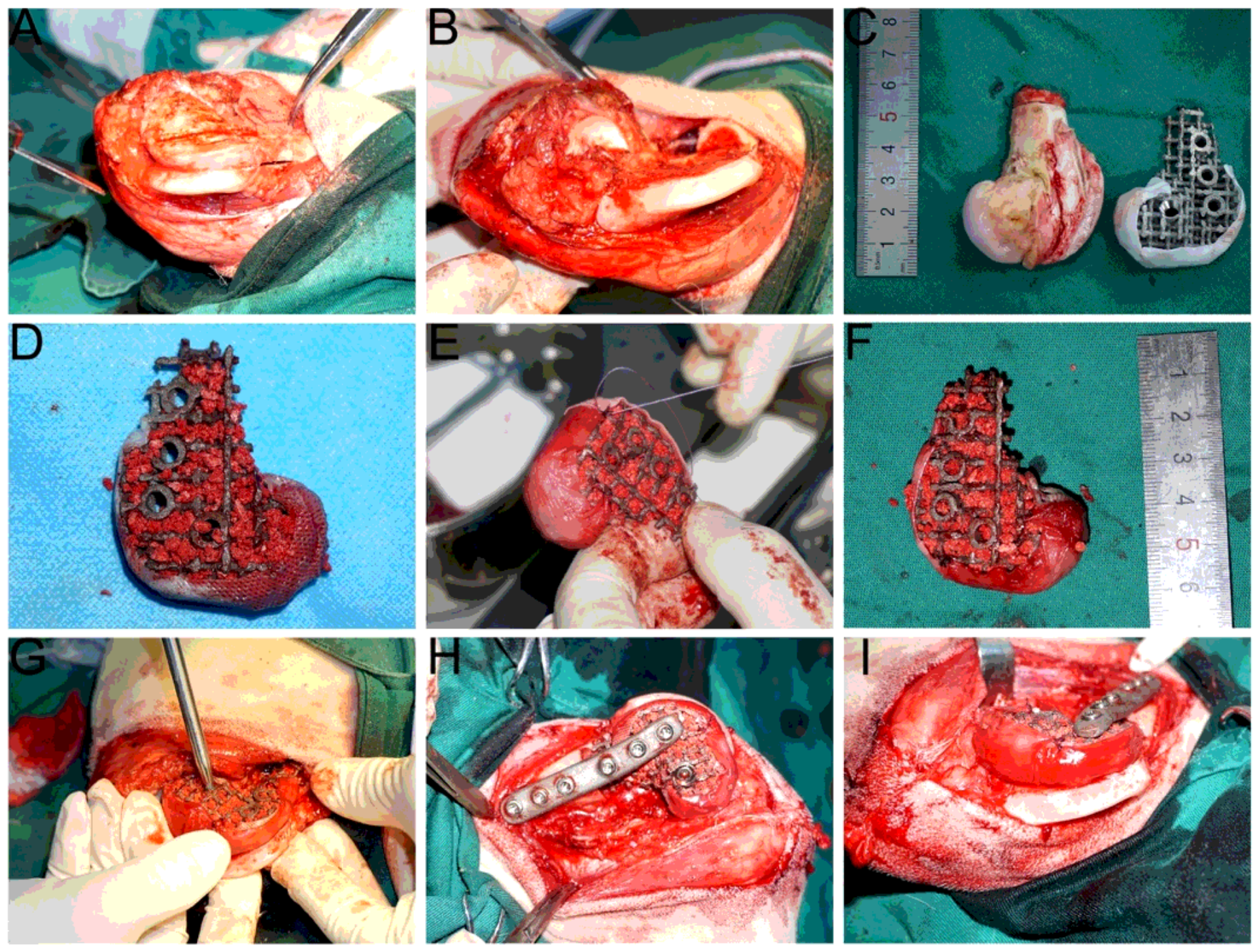

Figure 6

Establishment and repair of a goat bone defect model. $(a-c)$ The lateral half of the distal femur was removed to establish the bone defect model. $(d-f)$ MSCs/ $\beta$-TCP particles were filled into the Ti6AI4V frame, and a Bio-gide membrane was sutured into the PCL layer to round the distal surface. $(g-i)$ The prepared frame was fixed in the defect area. 


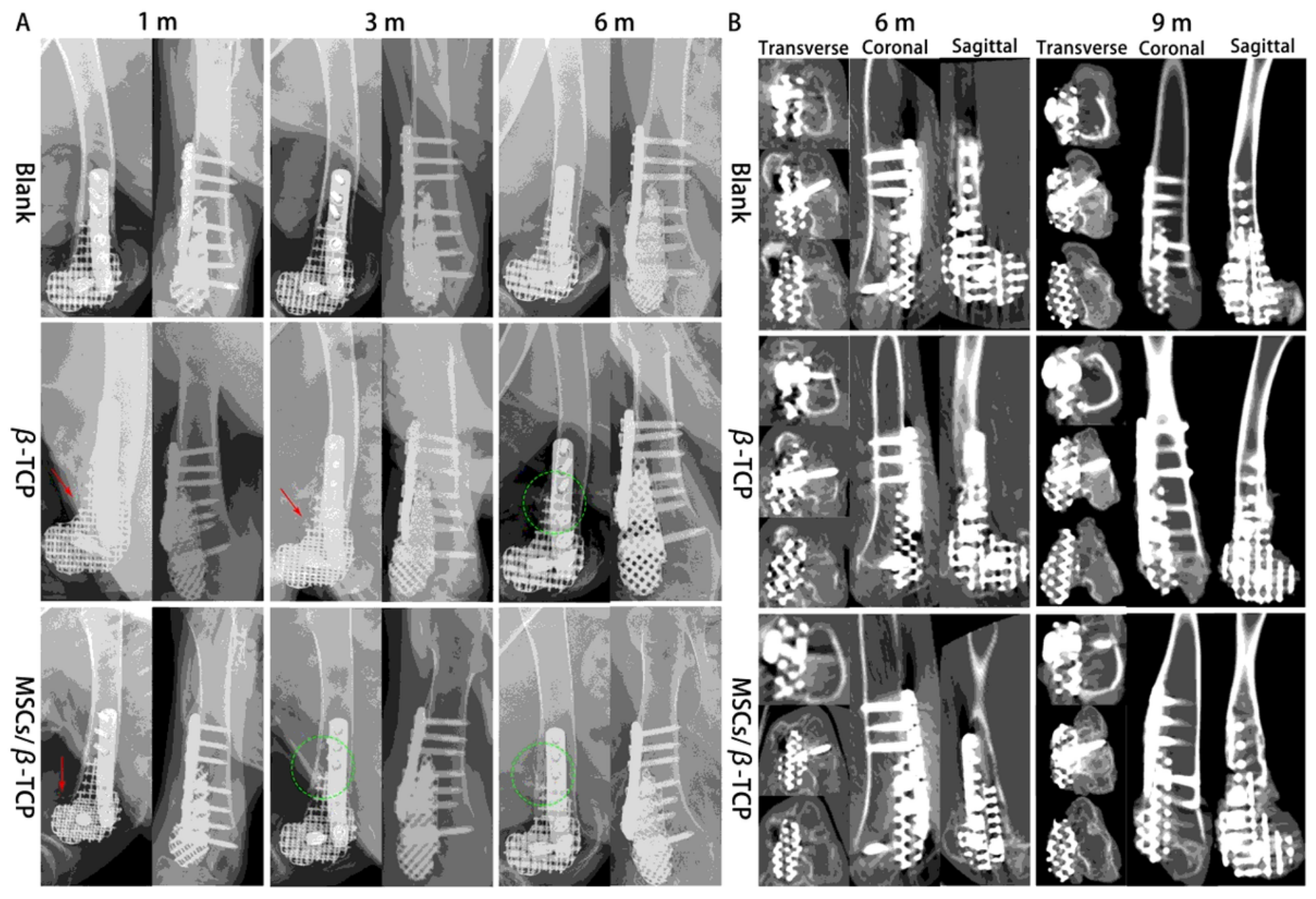

Figure 7

Evaluation of bone defect repair by imaging examinations. (a) X-ray radiography showed that the frames in each group matched the defect shape perfectly. The sign of particles around the frames (red arrow) is observed in the $\beta$-TCP group at 1 month and 3 months after surgery and in the MSCs/ $\beta$-TCP group only at 1 month after surgery. The bone hyperplasia sign between bone and frame (green circle) can be seen at 3 months after surgery in the MSCs/ $\beta$-TCP group, but till at 6 month after surgery in the $\beta$-TCP group. (b) CT image showing tissue growth inner frames in each group at 6 and 9 months after surgery. 
A

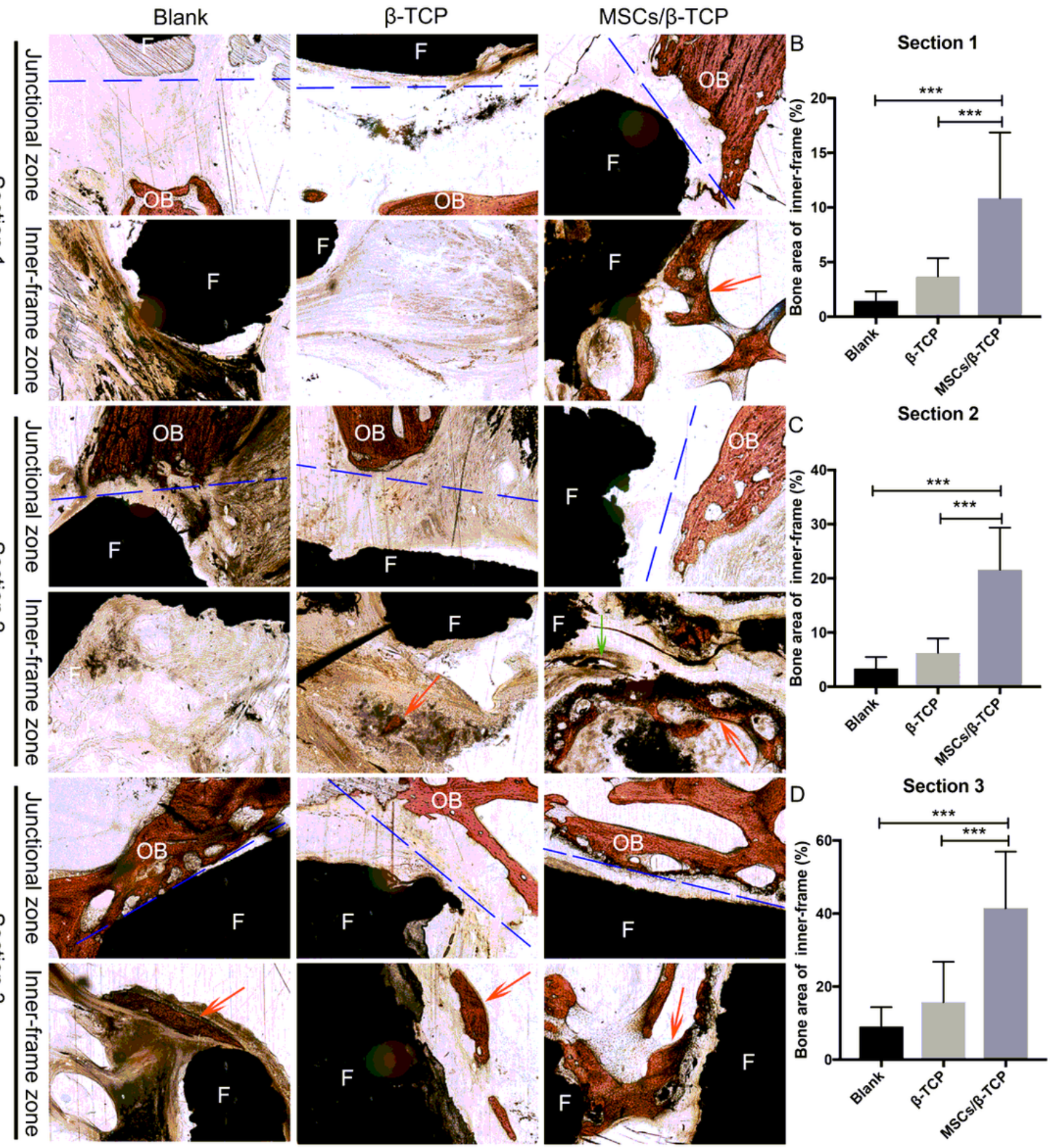

Figure 8

Histological evaluation of bone defect repair by Van Gieson's picro-fuchsin staining. (a) In the proximal section (section 1), obvious trabecular bone is seen in the inner frame zone in the MSCs/ $\beta$-TCP group despite suboptimal bone integration at the junctional site between the contralateral side and frame in each group. In the middle section (section 2), there is still no obvious sign of bone growth from the contralateral side at the junctional site in each group; however, new bone with the Haver's system has abundantly filled the inner frame in the MSCs/ $\beta$-TCP group, whereas there is limited new bone growth in the $\beta$-TCP group and almost no bone growth in the blank group. In the distal section (section 3 ), optional 
bone integration at the junctional site is seen in each group, but only in the MSCs/ $\beta$-TCP group formed new trabecular bone. $(b-d)$ Quantitative analysis of bone area within the frames can be seen in each section. (The blue dotted line represents the dividing line between the frame and offside bone; ' $F$ ' and 'OB' stand for frame and offside bone respectively and the 'Red arrow' and 'Green arrow' point to new bone and the Haver's system, respectively.) 